\title{
EchoGéo
}

$19 \mid 2012$

Du littoral à la haute mer : quelles recherches récentes en géographie?

\section{Les observatoires du trait de côte en France métropolitaine et dans les DOM}

\author{
Serge Suanez, Manuel Garcin, Thomas Bulteau, Mathias Rouan, Loïc \\ Lagadec et Laurence David
}

\section{OpenEdition}

\section{Journals}

Édition électronique

URL : https://journals.openedition.org/echogeo/12942

DOI : $10.4000 /$ echogeo. 12942

ISSN : 1963-1197

Éditeur

Pôle de recherche pour l'organisation et la diffusion de l'information géographique (CNRS UMR 8586)

Référence électronique

Serge Suanez, Manuel Garcin, Thomas Bulteau, Mathias Rouan, Loïc Lagadec et Laurence David, « Les observatoires du trait de côte en France métropolitaine et dans les DOM », EchoGéo [En ligne], 19 | 2012, mis en ligne le 10 février 2012, consulté le 10 août 2021. URL : http://journals.openedition.org/ echogeo/12942 ; DOI : https://doi.org/10.4000/echogeo.12942

Ce document a été généré automatiquement le 10 août 2021.

EchoGéo est mis à disposition selon les termes de la licence Creative Commons Attribution - Pas d'Utilisation Commerciale - Pas de Modification 4.0 International (CC BY-NC-ND) 


\title{
Les observatoires du trait de côte en France métropolitaine et dans les DOM
}

\author{
Serge Suanez, Manuel Garcin, Thomas Bulteau, Mathias Rouan, Loïc \\ Lagadec et Laurence David
}

\section{NOTE DE L'ÉDITEUR}

Certaines des illustrations ne sont pas très lisibles à l'écran aussi vous sont-elles proposées en format PDF pour impression à partir de l'onglet "annexe".

\section{Introduction}

1 Le rapport publié en 2004 par la Communauté Européenne indique qu'environ $20 \%$ des côtes européennes sont soumises à une sévère érosion, avec toutefois de grandes disparités suivant les pays (European Commission, 2004). $5 \%$ de ces rivages sont aujourd'hui protégés, mais pour autant, $15 \%$ de ce littoral artificialisé continuent de reculer avec pour conséquence une aggravation du risque lié à la submersion marine pour les côtes les plus basses.

2 A l'échelle de la France métropolitaine, le constat dressé par l'Ifen montre que le quart du linéaire côtier est en érosion (soit $1720 \mathrm{~km}$ sur $5500 \mathrm{~km}$ de côte), 44 \% sont stables et seulement $10 \%$ progradent (Colas, 2006). Le reste du littoral est constitué de côtes artificiellement figées (zones portuaires et digues) ou de côtes dont la dynamique n'est pas étudiée (respectivement $17 \%$ et $5 \%$ du linéaire côtier). Mais là encore, de fortes disparités existent en fonction de la morphologie (illustration 1a). Plus de $50 \%$ des littoraux sableux reculent car ils connaissent un déficit sédimentaire; l'érosion est beaucoup plus faible sur les côtes rocheuses généralement plus résistantes aux agents érosifs. Inversement, les côtes « vaseuses » regroupant les fonds de baie, d'estuaire, et 
les marais maritimes, progradent dans plus de $66 \%$ des cas, ce qui représente $55 \%$ de tout le linéaire côtier métropolitain.

3 L'étude de l'Ifen montre également de fortes disparités régionales ou départementales (illustration 1b). Ces différences sont là encore principalement liées au type de côte et à l'action anthropique sur le milieu naturel (extraction de matériaux dans les rivières ou en mer, barrages hydroélectriques, ouvrages portuaires et de défense littorale bloquant le transit, etc.). Ainsi, les littoraux du nord de la France de la frontière belge au Calvados sont les plus érodés ( $85 \%$ du littoral du Pas-de-Calais et $92 \%$ de la SeineMaritime). Les seuls secteurs épargnés sont les fonds de baies et estuaires (la Seine, la Canche, l'Authie ou la Somme). Inversement plus de la moitié du linéaire côtier de Bretagne et de Loire-Atlantique est stable, cette part étant maximale dans le Morbihan (71\%). L'érosion redevient importante sur le littoral atlantique, au sud de l'embouchure de la Loire. Elle touche plus de $52 \%$ du linéaire côtier. Seuls les marais maritimes de la baie de Bourgneuf, le Marais poitevin, le bassin d'Arcachon et quelques portions du littoral landais sont épargnés. Le littoral méditerranéen enfin montre des situations très contrastées entre le littoralcorse essentiellement rocheux, et majoritairement stable, et les littoraux sableux languedociens et du delta du Rhône, où l'érosion peut être importante à certains endroits. Plus à l'est, on note unegrande hétérogénéité des types de côtes. L'érosion y esttrès variable suivant que l'on se trouve sur les falaises rocheuses des calanques de Provence et des massifs cristallins du Var et des Alpes Maritimes, ou sur les cordons sableux de la presqu'île de Giens et de Fréjus.

Cette érosion présente un risque notable car environ $23 \%$ des zones urbanisées se situent à moins de $250 \mathrm{~m}$ des côtes soumises à un recul. L'érosion peut donc y revêtir un enjeu majeur d'autant plus que l'urbanisation continue à croître en bord de mer (10\% des surfaces bâties se concentrent sur $4 \%$ du territoire des communes littorales).

Illustration 1a - Linéaire côtier en érosion, stable ou progradant en fonction du type rocheux, sableux ou vaseux de côtes. 1b - Part du linéaire côtier en érosion, stable ou progradant par départements littoraux

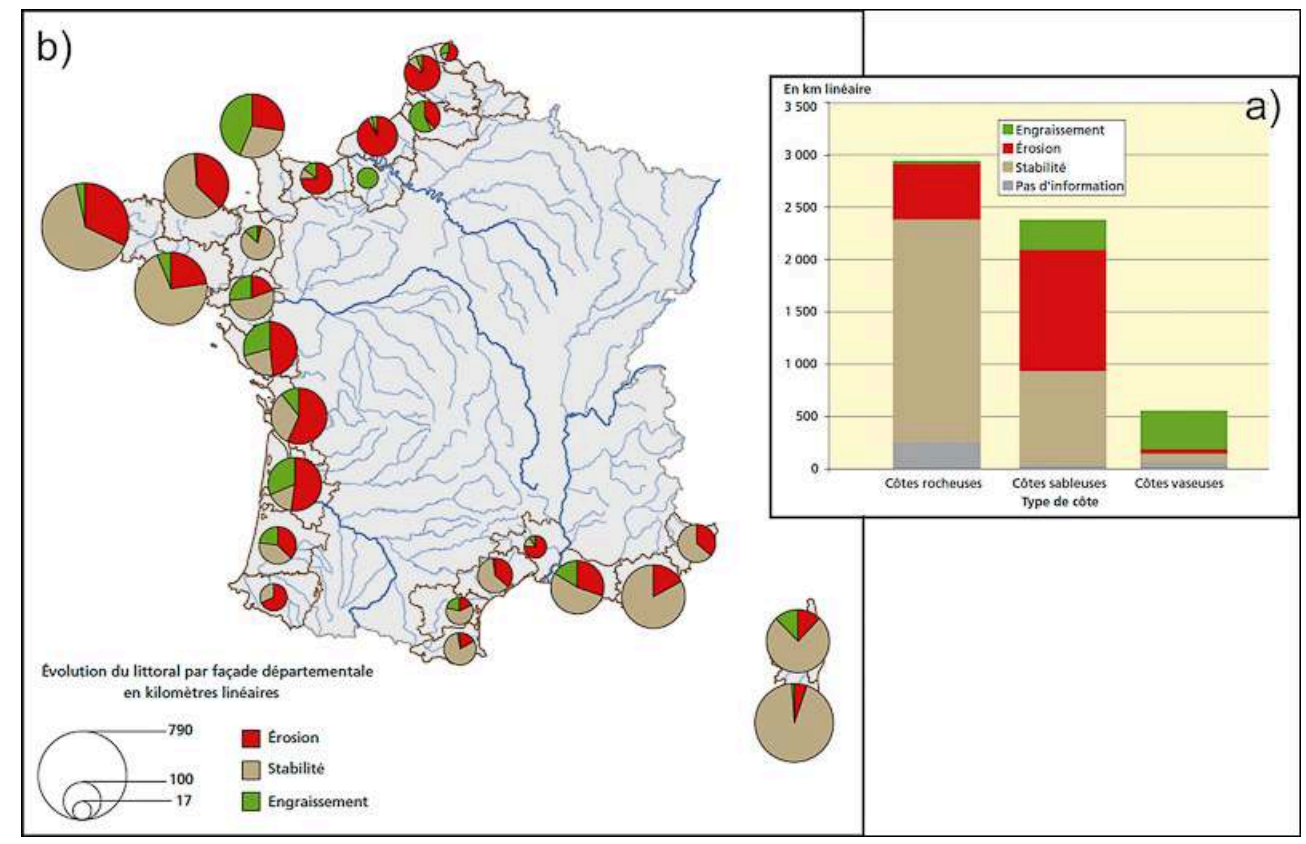

Sources : Données Ifen sur l'érosion du littoral français métropolitain ; S. Colas, 2006. 
Face à ce constat, l'observation du trait de côte est devenu un enjeu essentiel dans la gestion des risques littoraux et la maîtrise des aléas car ces suivis fournissent des éléments d'aide à la décision pour la défense côtière et l'aménagement littoral (Ruggiero et al., 2000 ; Rieb et Walker, 2001 ; Hamm et al., 2002 ; Meur-Férec et al., 2008). Bien plus encore, la réussite des politiques de gestion intégrée de la zone côtière (GIZC) doit aujourd'hui tenir compte des processus physiques dont la compréhension repose sur l'acquisition de longues séries d'observations environnementales (Solomon et Forbes, 1999). A titre d'exemple, le suivi de la cinématique du trait de côte le long de la côte est des Etats-Unis a permis de fournir les informations nécessaires à la mise en place des politiques de gestion de l'érosion côtière par le FEMA (Federal Emergency Management Administration) (Leatherman, 2003). De même, la base de données JARKUS élaborée par le Dutch Department of Public Works (Rijkswaterstaat), et qui compile plus de 30 années de mesures de profil de plage le long du littoral néerlandais a permis de mieux comprendre l'évolution spatio-temporelle de ce dernier (Louisse et van der Meulen, 1991), et de définir une politique nationale de préservation de la ligne de rivage pouvant recourir à d'importants rechargements de plage lorsque nécessaire (Koster et Hillen, 1995). Les suivis du trait de côte peuvent également être réalisés au titre de la recherche fondamentale. C'est l'exemple du programme DUCK (Displaying Underwater Conditions Kinematically) - North Carolina, entrepris depuis 1981 par le corps d'ingénieurs de l'armée américaine (US Army Corps of Engineers Field Research Facility), et qui consiste en des mesures bi-hebdomadaires de profil transversal de plage utilisant un CRAB (Coastal Research Amphibious Buggy) (Mason, 1985 ; Howd et Birkemeier, 1987 ; Lee et Birkemeier, 1993). Ces données ont permis de faire avancer la connaissance théorique dans des champs thématiques aussi variés que l'évolution à long terme du profil de plage, la migration des corps sédimentaires (barres) en milieu infratidal, la question de la profondeur de fermeture (closure depth) ou des phénomènes de setup et runup, la modélisation des processus hydrosédimentaires (Birkemeier, 1985 ; Larson et Kraus, 1994 ; Reeve et al., 2007 ; Southgate, 2008).

6 L'objectif de cet article est de faire dans un premier temps l'inventaire des différents observatoires du trait de côte en France métropolitaine et dans les DOM, à une échelle régionale et infra-régionale. Ce travail de synthèse a été réalisé par le BRGM à la demande du MEEDDM (Bulteau et al., 2011), comme un préalable à l'engagement $74 \mathrm{du}$ Grenelle de la Mer : "développer une méthodologie et une stratégie nationale pour la gestion du trait de côte, pour le recul stratégique et la défense contre la mer ». Dans un second temps, nous traitons d'un exemple en présentant les travaux d'observation du trait de côte réalisé en Bretagne dans le cadre de l'observatoire du Domaine Côtier (ODC) de l'Institut Universitaire Européen de la Mer (IUEM), et de l'élaboration d'une base de données interactive appelée MADDOG (Mise A Disposition de Données d'Observation Géomorphologique).

\section{Inventaire et classification des observatoires du trait de côte français}

\section{Méthode de recensement}

Le travail d'inventaire a consisté dans un premier temps à recenser les observatoires et/ou les réseaux d'observation du trait de côte à l'échelle du territoire 
métropolitain et des DOM (Bulteau et al., 2011). Par "observatoire ", nous entendons une structure qui acquiert des données relatives au trait de côte, de manière plus ou moins fréquente, et sur une zone plus ou moins étendue, avec pour objectif principal d'analyser les évolutions temporelles de ce dernier. Dans un second temps, des entretiens avec les responsables de ces observatoires ont permis de préciser leurs finalités, leurs modes de fonctionnement (types et fréquences d'acquisition, analyses et valorisation des données, etc.), leurs sources de financement, et leurs liens avec les organismes et/ou les collectivités. Le recensement a été effectué en 2010 et a montré l'existence de 52 «opérations » de suivi du trait de côte dont les spécificités varient énormément suivant les types d'observation et les façades maritimes. Cette diversité des situations nous a obligés à élaborer une typologie reposant sur des critères tels que la fréquence des mesures, les conditions de déclenchement des mesures, les modalités de mise à jour des données, l'échelle spatiale, le type de valorisation ou d'utilisation des données. Ces critères nous ont conduits à définir cinq types d'observatoire regroupés en trois catégories (tableau 1).

Tableau 1 - Typologie des observatoires de suivi du trait de côte

\begin{tabular}{|c|c|c|c|c|c|c|}
\hline Type & Catégorie & Fréquence & $\begin{array}{c}\text { Valorisation ou } \\
\text { utilisation }\end{array}$ & Echelle spatiale & Déclenchement & Mise à jour \\
\hline 1 & \multirow{2}{*}{ 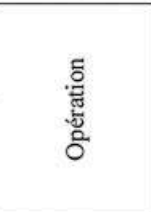 } & $\begin{array}{l}\text { Mesures période } \\
\text { unique, étude } \\
\text { ponctuelle }\end{array}$ & $\begin{array}{l}\text { Projet } \\
\text { d'aménagements } \\
\text { littoraux, risque }\end{array}$ & Réduite (locale) & Ponctuel & $\begin{array}{l}\text { Opportuniste } \\
\text { voire jamais }\end{array}$ \\
\hline 2 & & $\begin{array}{l}\text { Mesures multi- } \\
\text { dates, non planifié }\end{array}$ & $\begin{array}{l}\text { Aménagements } \\
\text { littoraux, risque, } \\
\text { activité de } \\
\text { recherche }\end{array}$ & Réduite (locale) & Ponctuel & Opportuniste \\
\hline 3 & \multirow{2}{*}{ 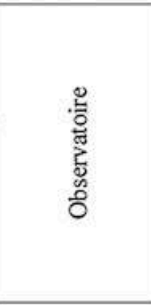 } & $\begin{array}{l}\text { Suivi pluriannuel } \\
\text { de courte durée / } \\
\text { plutôt mono- } \\
\text { paramètre }\end{array}$ & $\begin{array}{c}\text { Activité de } \\
\text { recherche, aide à } \\
\text { la décision }\end{array}$ & $\begin{array}{l}\text { Plusieurs } \\
\text { communes, } \\
\text { département }\end{array}$ & Programmé & $\begin{array}{l}\text { Fréquence } \\
\text { programmée }\end{array}$ \\
\hline 4 & & $\begin{array}{l}\text { Observatoires } \\
\text { pérennes (longue } \\
\text { durée de vie, multi } \\
\text { paramètres) }\end{array}$ & $\begin{array}{l}\text { Aide à la } \\
\text { décision, bases } \\
\text { de données, } \\
\text { activité de } \\
\text { recherche }\end{array}$ & $\begin{array}{l}\text { Département(s), } \\
\text { région }\end{array}$ & Programmé & $\begin{array}{l}\text { Fréquence } \\
\text { programmée } \\
\text { et action si } \\
\text { évènement } \\
\text { exceptionnel } \\
\text { (tempête) }\end{array}$ \\
\hline SIL & $\begin{array}{c}\text { Méta- } \\
\text { observatoire }\end{array}$ & Pas d'acquisition & $\begin{array}{l}\text { Suivi du littoral } \\
\text { (indicateurs } \\
\text { autres que le trait } \\
\text { de côte) }\end{array}$ & $\begin{array}{c}\text { Régionale à } \\
\text { nationale }\end{array}$ & $\begin{array}{c}\text { Pas } \\
\text { d'acquisition } \\
\text { propre }\end{array}$ & $\begin{array}{c}\text { Fonction des } \\
\text { accords avec } \\
\text { les } \\
\text { fournisseurs } \\
\text { de données }\end{array}$ \\
\hline
\end{tabular}

Source : Bulteau et al., 2011

\section{Typologie des observatoires français}

Les observatoires de type 1 s'inscrivent dans une logique d'acquisition ponctuelle de connaissances pour une gestion à court terme du littoral. On peut distinguer deux sortes d'observatoire de type 1 en fonction de leurs objectifs : (i) répondre à un besoin local d'une collectivité ou d'un ensemble de partenaires, (ii) faire un état des lieux du littoral sur une zone précise à un instant donné. Dans les deux cas, c'est une action immédiate qui est envisagée. A titre d'exemple on peut citer les études ponctuelles commanditées par les Conseils généraux de la région Provence-Alpes-Côte-d'Azur (PACA) en 2004 pour le Var, (IN VIVO, 2004), en 2007 pour les Alpes-Maritimes, (BCEOM, 2007), et en 2008 pour les Bouches-du-Rhône, (SAFEGE, 2008). 
9 Les observatoires de type 2 ont des activités de suivi du trait de côte liées par exemple à un programme de recherche mais sans qu'il ait ni une périodicité et ni une pérennisation de l'acquisition. C'est le cas par exemple des actions de suivi effectuées par le Laboratoire Océanographie et Géosciences de l'Université du Littoral Côte d'Opale.

10 Les observatoires de type 3 reposent sur des campagnes de mesures périodiques et pérennes couvrant une zone géographique rarement plus étendue que le département. A la différence des observatoires de type 1 et 2 , ces suivis ne sont pas réalisés afin de répondre à une demande sociétale ponctuelle ou à un projet de recherche limité dans le temps. C'est le cas du suivi réalisé par la Direction Départementale des Territoires et de la Mer (DDTM) de la Somme sur les 70 kilomètres de côte du département ou de l'ODC IUEM dans le Finistère, qui sera présenté ultérieurement.

11 Les observatoires de type 4 collectent des données sur une zone étendue couvrant généralement la région, de manière régulière et pérenne. Ils analysent les données, les interprètent et les rendent accessibles. Ils peuvent par ailleurs réaliser des expertises pour des collectivités locales ou des organismes; c'est le cas par exemple de l'Observatoire de la Côte Aquitaine (OCA) dont le maître d'œuvre principal est le BRGM.

12 Les observatoires de type 3 et 4 représentent les acteurs majeurs du suivi du trait de côte. La fréquence des acquisitions de données est programmée même si elle peut varier entre les observatoires. Ces données permettent de retracer l'évolution morphologique du littoral à partir de la cinématique du trait de côte, des changements de profils de plage, ou du calcul de bilans sédimentaires en 3D et de volumes des éboulements pour les côtes à falaise, etc. Certains de ces observatoires s'intéressent également aux processus hydrosédimentaires et acquièrent dans ce but des données bathymétriques et des données relatives aux forçages hydrodynamiques (marée, vagues et courants).

13 Enfin, les Systèmes d'Information sur le Littoral (SILs) comme BOSCO (Base d'Observations pour le Suivi des CÔtes), Géolittoral et l'observatoire du littoral du SOeS (ex Ifen) sont individualisés. Les SILs n'acquièrent pas de nouvelles données mais capitalisent et/ou les traitent de façon à produire des documents d'analyse et de synthèse compréhensibles et utiles à un très large public tel que des scientifiques, des techniciens et gestionnaires du territoire, ou le simple usager (Bersani et al., 2006).

La répartition de ces différents types d'observatoires le long du littoral métropolitain et des DOM montre une grande hétérogénéité spatiale (tableau 2). Les suivis ponctuels correspondant aux observatoires de type 1 et 2 sont les plus répandus; ils pourraient toutefois être bien plus nombreux car le recensement de ces derniers n'est pas exhaustif. En effet, de nombreuses opérations de suivi sur du court terme n'ont certainement pas été déclarées et/ou inventoriées par tous les acteurs. A l'inverse il existe très peu d'observatoires de type 4.

15 Les entretiens ont montré que les observatoires pérennes de type 4 étaient principalement présents dans les régions confrontées à des problèmes d'érosion importants comme c'est le cas dans le Languedoc-Roussillon ou en Aquitaine. Par exemple, l'observatoire de la Côte Aquitaine a été initié dans le cadre d'un Contrat de Plan État-Région (CPER) entre 1996 et 2000, associant le Conseil Régional d'Aquitaine, le BRGM et l'IFREMER (Institut français de recherche pour l'exploitation de la Mer). Ce premier CPER a été prolongé en 2001-2006, et en 2007-2013 (Favennec et Mallet, 2008 ; 
Mugica et al., 2009 ; Aubié et al., 2011). A ces deux autres CPER, le nombre de partenaires s'est accru en intégrant notamment les Conseils généraux et les Syndicats intercommunaux. A l'heure actuelle, l'OCA bénéficie également de fonds FEDER (Fonds Européen de Développement Régional).

A l'inverse, là où le recul de la côte n'apparaît pas comme un problème majeur à l'échelle régionale, comme c'est le cas notamment pour tout le littoral rocheux de la Côte d'Azur en région PACA, ce sont essentiellement des suivis ponctuels de type 1 et 2 qui ont été réalisés.

Tableau 2 - Nombre d'observatoires par façade littorale régionale et par type

\begin{tabular}{|c|c|c|c|c|c|c|}
\hline \multirow{9}{*}{ Manche-Mer du Nord-Atlantique } & \begin{tabular}{|r|} 
Région \\
\end{tabular} & Type 1 & Type 2 & Type 3 & Type 4 & Total Région \\
\hline & Nord Pas de Calais & 1 & 1 & 2 & & 4 \\
\hline & Picardie & 1 & 2 & 1 & & 4 \\
\hline & Normandie & 1 & 2 & 1 & 1 & 5 \\
\hline & Trans régional Normandie / Picardie & & & & 1 & 1 \\
\hline & Bretagne & 2 & 1 & 2 & & 5 \\
\hline & Pays de la Loire & 2 & 3 & 2 & & 7 \\
\hline & Poitou Charentes & 1 & & 2 & & 3 \\
\hline & Aquitaine & & 1 & & 1 & 2 \\
\hline & & & & & & \\
\hline \multirow{3}{*}{ Méditerranée } & Languedoc Roussillon & & 1 & 3 & 1 & 5 \\
\hline & PACA & 4 & 1 & 2 & & 7 \\
\hline & Corse & & & & 1 & 1 \\
\hline & & & & & & \\
\hline \multirow{2}{*}{ Océan Indien } & Réunion & & & 2 & & 2 \\
\hline & Mayotte & & 1 & & & 1 \\
\hline \multirow{5}{*}{ Atlantique Caraibes } & & & & & & \\
\hline & Guyane & 1 & 2 & & & 3 \\
\hline & Martinique & 1 & & & & 1 \\
\hline & Guadeloupe & 1 & & & & 1 \\
\hline & Total par type & 15 & 15 & 17 & 5 & 52 \\
\hline
\end{tabular}

Source : Bulteau et al., 2011

La répartition spatiale des observatoires par région pour le territoire français métropolitain (tous types confondus) pourrait laisser croire que le littoral de métropole est entièrement suivi (illustration 2). Dans la réalité, la situation est beaucoup plus complexe car certains acteurs ont des zones de compétence étendues mais ne font des suivis que sur des espaces restreints; c'est le cas par exemple de la DDTM (Direction Départementale des Territoires Maritimes) du Pas-de-Calais sur les zones les plus sensibles du littoral de la Mer du Nord ou de la DDTM de la Seine-Maritime sur les ouvrages de défense et les cordons de galets, notamment dans le secteur de Cayeux-surMer.

La situation est similaire dans les DOM où l'on comptabilise huit observatoires répartis entre les îles de la Réunion, de la Guadeloupe et de la Martinique, de Mayotte, de la Guyane française (illustration 3). A l'exception de l'île de la Réunion où l'on trouve deux observatoires de type 3 qui sont gérés par le BRGM et le laboratoire des GéoSciences de l'université de la Réunion, pour les autres DOM, les suivis du trait de côte reposent essentiellement sur des actions ponctuelles. Ces dernières concernent des études visant à faire un état des lieux très général sur l'évolution historique du trait de côte, et/ou à mesurer à un instant " $\mathrm{T}$ " l'impact d'un évènement extrême sur le littoral (Lachassagne et Paulin, 1990 ; Barras et Koller, 2010). De façon générale, la couverture spatiale de ces observatoires dans les DOM apparait très discontinue, seuls quelques sites sont suivis plus ou moins régulièrement. Ceci étant, soulignons toutefois que des projets de mise en place d'observatoires de type 3 ou 4 sont actuellement en cours. C'est le cas notamment pour la Guyane, la Guadeloupe et la Martinique dans le cadre du 
projet CARIBSAT ${ }^{1}$ financé par l'Europe au sein du programme Interreg IV Caraïbe ${ }^{2}$, dont une des actions porte sur l'évolution et la "vulnérabilité du trait de côte ». C'est également le cas pour la Réunion avec le lancement d'un SOERE suivi de plage dans le cadre de l'OSU-Réunion.

Illustration 2 - Localisation des différents types d'observatoires du trait de côte pour le littoral français métropolitain

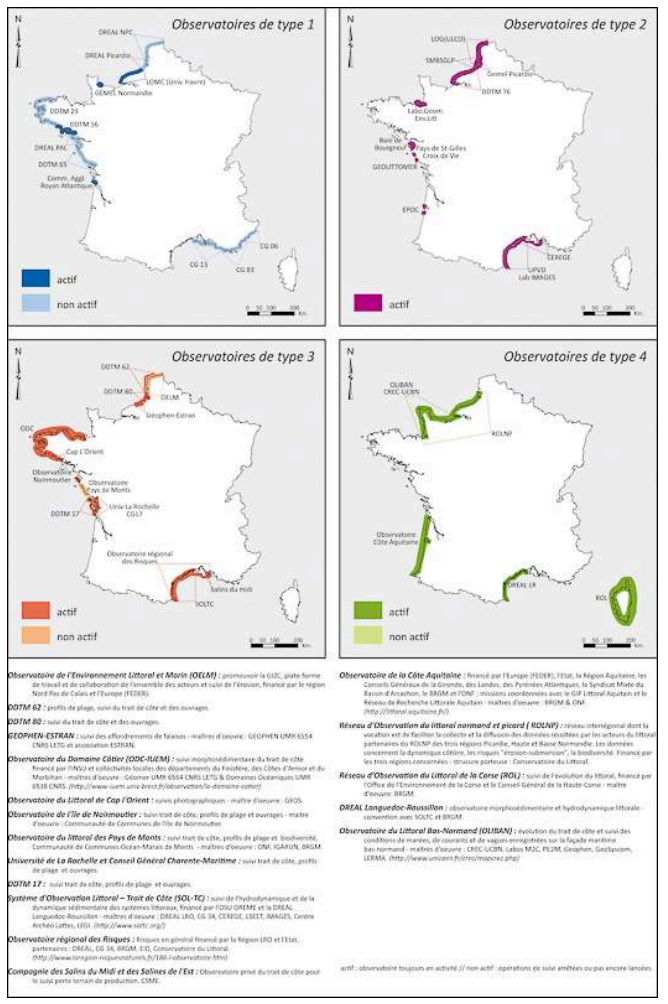


Illustration 3 - Localisation des différents types d'observatoires du trait de côte pour le littoral des Départements d'Outre Mer (DOM)

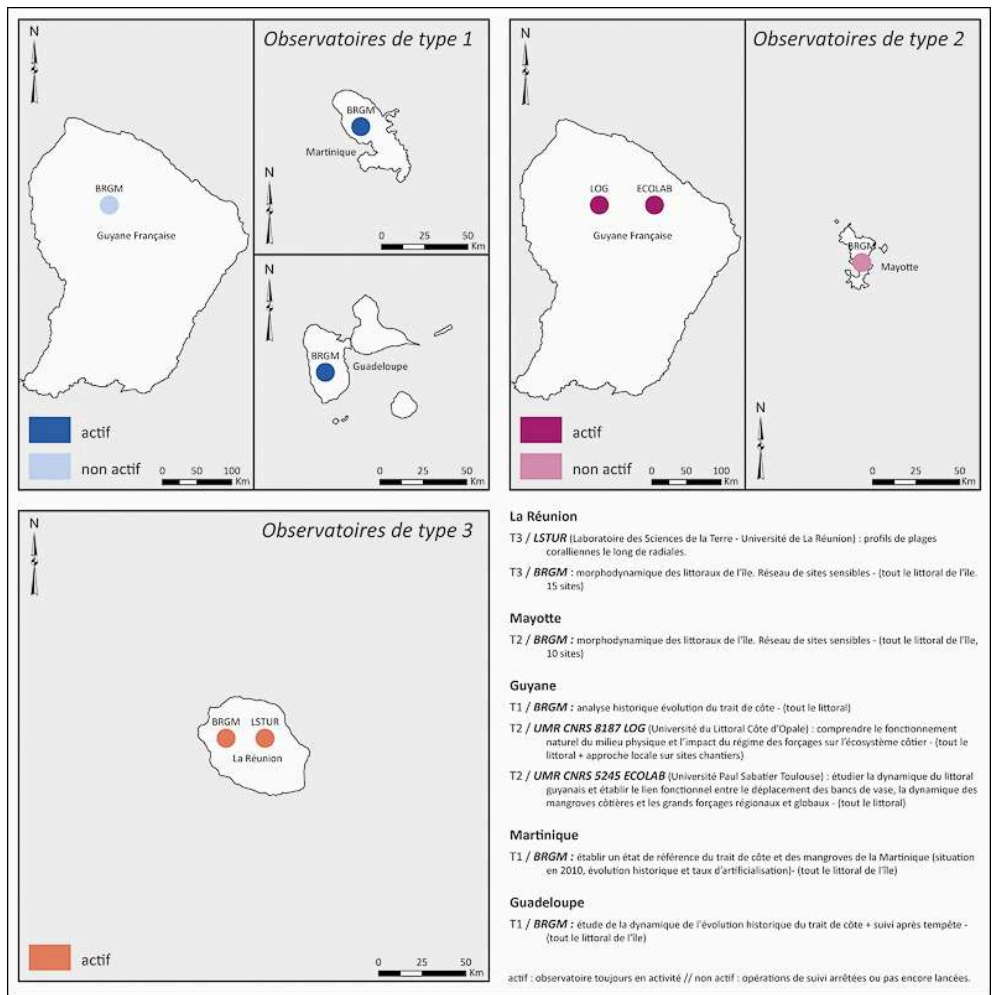

\section{Diversité des maîtres d'œuvre}

La gestion de ces observatoires montre également une grande diversité des maîtres d'œuvre. A l'exception des observatoires de type 1 dont les suivis ponctuels sont le plus souvent réalisés par des bureaux d'études, tous les autres types d'observatoires sont gérés par :

- les services de l'Etat, tels que les Directions Régionales de l'Environnement, de l'Aménagement et du Logement (DREAL) ou les DDTM (DREAL : Nord Pas-de-Calais, Picardie Basse-Normandie, Languedoc-Roussillon, etc.) ; DDTM : Nord Pas-de-Calais, Somme, Seine Maritime, Loire Atlantique, Charente-Maritime);

- les centres et équipes de recherches universitaires : Institut Universitaire Européen de la Mer, laboratoire GEOPHEN (Géographie Physique et Environnement), CREC M2C (Centre de Recherches en Environnement Côtier-Morpho-dynamique Continentale et Côtière), laboratoire LIENSs (LIttoral ENvironnement et Sociétés), laboratoire EPOC (Environnements et Paléoenvironnements Océaniques), laboratoire IMAGES (Institut de Modélisation et d'Analyse en Géo-Environnement et Santé), CEREGE (Centre Européen de Recherche et d'Enseignement des Géosciences de l'Environnement), laboratoire ECOLAB (Ecologie Fonctionnelle), laboratoire LOG (Laboratoire Océanographie et Géosciences ;

- les collectivités : Régions, Conseils généraux, Communautés d'agglomération, communes ;

- les établissements publics : Conservatoire du Littoral, BRGM, IFREMER, ONF (Office National des Forêts) ;

- les bureaux d'études : SOGREAH (Société Grenobloise d'Etudes et d'Applications Hydrauliques), DHI (Danish Hydraulic Institute), GEOS (Aménagement/Environnement Littoral) ; 
- les structures associatives : GEMEL (Groupe d'Etude des Milieux Estuariens et Littoraux)

de Normandie et de Picardie ;

- les sociétés privées : la Compagnie des Salins du Midi et des Salines de l'Est pour les secteurs des salins de Giraud et d'Aigues-Mortes en Camargue. œuvre dans l'observation, et la finalité même des suivis. Les centres de recherches universitaires par exemple effectuent souvent des suivis pour répondre à des problématiques scientifiques et n'ont pas vocation à faire de l'observation opérationnelle au sens où les mesures sont régulières, pérennes, et orientées vers la constitution d'une base de données. Il s'agit avant tout de faire progresser la connaissance théorique des processus côtiers. Ceci explique en partie que les suivis réalisés par ce type de maître d'œuvre ne concernent que des zones géographiques souvent restreintes (échelle de la plage) en ayant recours à des techniques de mesure expérimentales parfois très lourdes à mettre en œuvre (utilisation de drone ou de lidar terrestre). Il y a toutefois des exceptions, notamment lorsque les centres de recherche universitaires arrivent à financer une partie de leurs suivis par des collectivités dans le cadre de la recherche contractuelle. C'est le cas de l'observatoire du Domaine Côtier IUEM. Dans ce cas, le suivi du trait de côte peut être étendu à des aires géographiques plus vastes englobant l'ensemble du département.

21 A l'inverse, lorsque la maîtrise d'oeuvre est donnée aux services de l'Etat (services maritimes, DDTM, etc.), l'observation est effectuée principalement dans un but opérationnel et repose généralement sur des méthodes classiques de mesures de profils de plage ou de trait de côte au DGPS. Il s'agit avant tout de réaliser une "surveillance " de la zone littorale sans que les données ne soient valorisées d'un point de vue scientifique.

\section{Acquisition, archivage et interopérabilité des métadonnées}

\section{Diversité des méthodes de mesure et de stockage des données}

L'observation du trait de côte peut rapidement impliquer l'acquisition d'une grande variété de données en fonction du type de suivi (données géomorphologiques et sédimentaires ou relatives aux processus hydrosédimentaires), de la morphologie du site (différents outils peuvent être employés en fonction de l'accessibilité, de l'étendue de la zone d'étude, du type de côte, etc.), et de la dynamique du littoral (évolution rapide ou au contraire côte relativement stable).

Même si la plupart des observatoires utilisent des outils qui récupèrent des données géoréférencées dans un système de coordonnées connu, certains maitres d'œuvre ont recours à des appareillages tels que les tachéomètres ou les niveaux à bulle qui ne raccordent pas leurs mesures à un référentiel géodésique. Ces données (en coordonnées relatives) sont donc inexploitables en dehors de leur zone d'acquisition et ne peuvent être comparées qu'entre elles; c'est le cas du suivi de plage réalisé par la compagnie des Salins du Midi et des Salines de l'Est dans le delta du Rhône.

Les protocoles de mesure doivent également respecter certaines règles de manière à récupérer des données qui puissent être comparées entre elles d'une date sur l'autre. 
Pour les profils de plage par exemple, il est important que ces derniers soient calés à partir d'un repère fixe généralement appelé "tête de profil»; il est aussi important que l'azimut des radiales de profil reste le même à chaque campagne de levé. Lorsque ces règles ne sont pas respectées, l'analyse diachronique peut révéler des changements morphologiques qui ne sont alors que le résultat de l'erreur qui entache la mesure (Suanez et al., 2008).

Le mode de stockage diffère également selon les observatoires La plupart du temps, les données sont stockées sous format numérique mais il arrive qu'elles soient toujours sous format papier pour les données les plus anciennes; c'est le cas par exemple des mesures marégraphiques du Golfe du Lion dont la DREAL Languedoc-Roussillon est propriétaire. La numérisation des données papier permet de les rendre pérennes et plus facilement exploitables en vue de comparaisons avec des données plus récentes. Notons néanmoins la difficulté de comparer des données qui ont été acquises avec des outils et des protocoles de mesure différents (problèmes de précision de la mesure, de définition de l'objet de mesure, de calage géodésique de la donnée, etc.).

\section{Interopérabilité des métadonnées et visibilité des observatoires}

L'interopérabilité des métadonnées, lorsqu'elles existent, n'est pas assurée dans la majorité des cas bien qu'elle soit préconisée par la directive INSPIRE ${ }^{3}$. Cet outil réglementaire existe depuis 2007, et vise à favoriser l'échange des données au sein de la Communauté européenne dans le domaine de l'environnement pris dans un sens large. La difficulté à appliquer la directive INSPIRE est principalement due au fait que chaque observatoire fonctionne indépendamment des autres, et qu'il n'y a de ce fait aucune synergie fédératrice entre ces opérations de suivi. Les enquêtes ont montré que jusqu'à présent, le besoin d'interopérabilité n'est pas une priorité et/ou finalité exprimée par les différents maîtres d'oeuvre. Sur la totalité des observatoires recensés, seulement huit ont élaboré une base de métadonnées interopérables: GEMEL Picardie, Observatoire du Littoral des Pays de Monts, Observatoire de la Côte Aquitaine, Réseau d'Observation du Littoral (Corse), Réseau d'Observation du Littoral Normand-Picard, DREAL Languedoc-Roussillon, Observatoire Régional des Risques en LanguedocRoussillon, CEREGE.

Outre l'interopérabilité des métadonnées, la directive INSPIRE porte sur les données proprement dites et stipule qu'il doit être possible de combiner les données provenant de différentes sources de manière cohérente et de les partager entre plusieurs utilisateurs et applications.

S'agissant enfin, de la visibilité et de la communication des observatoires, à quelques exceptions près (GEMEL Picardie de type 2), seuls les observatoires de type 3 ou 4 possèdent un accès public via un site internet (Observatoire de la Côte Aquitaine, OLIBAN - Observatoire duLittoralBas-Normand, Réserve Naturelle de Camargue, etc.). En dehors de cet affichage qui s'adresse finalement à une communauté très restreinte (scientifiques, acteurs territoriaux, collectivités), il faut signaler que le grand public est dans l'ensemble très mal informé des actions de suivi du trait de côte et de l'importance que celui-ci revêt pour la mise en œuvre de stratégies de gestion efficaces et durables du littoral. 


\section{Étude de cas : Observatoire du Domaine Côtier de I'IUEM}

L'observatoire du domaine côtier (ODC) a été lancé en 2002 par l'IUEM de l'université de Brest. Il regroupe une dizaine de séries d'observation allant du domaine côtier au domaine hauturier ; certaines de ces séries ont par ailleurs été labellisées «OSU » en 2005 lorsque l'IUEM a été reconnu comme Observatoire des Sciences de l'Univers ${ }^{4}$. Le travail de suivi du trait de côte que nous présentons dans cette partie représente un élément de cet observatoire identifié sous le label « observatoire morphosédimentaire trait de côte ». Il concerne essentiellement les côtes d'accumulation constituées des cordons de galets et des plages de sables, et dans une moindre mesure, les falaises meubles (Dehouck, 2004 ; Suanez et Fichaut, 2005 ; Suanez et al., 2010). Cela représente à l'heure actuelle une quarantaine de sites répartis sur les trois départements bretons du Finistère, des Côtes d'Armor et du Morbihan (illustration 4).

Illustration 4 - Localisation des sites de mesures topo-morphologiques de l'ODC-IUEM pour l'observation du trait de côte

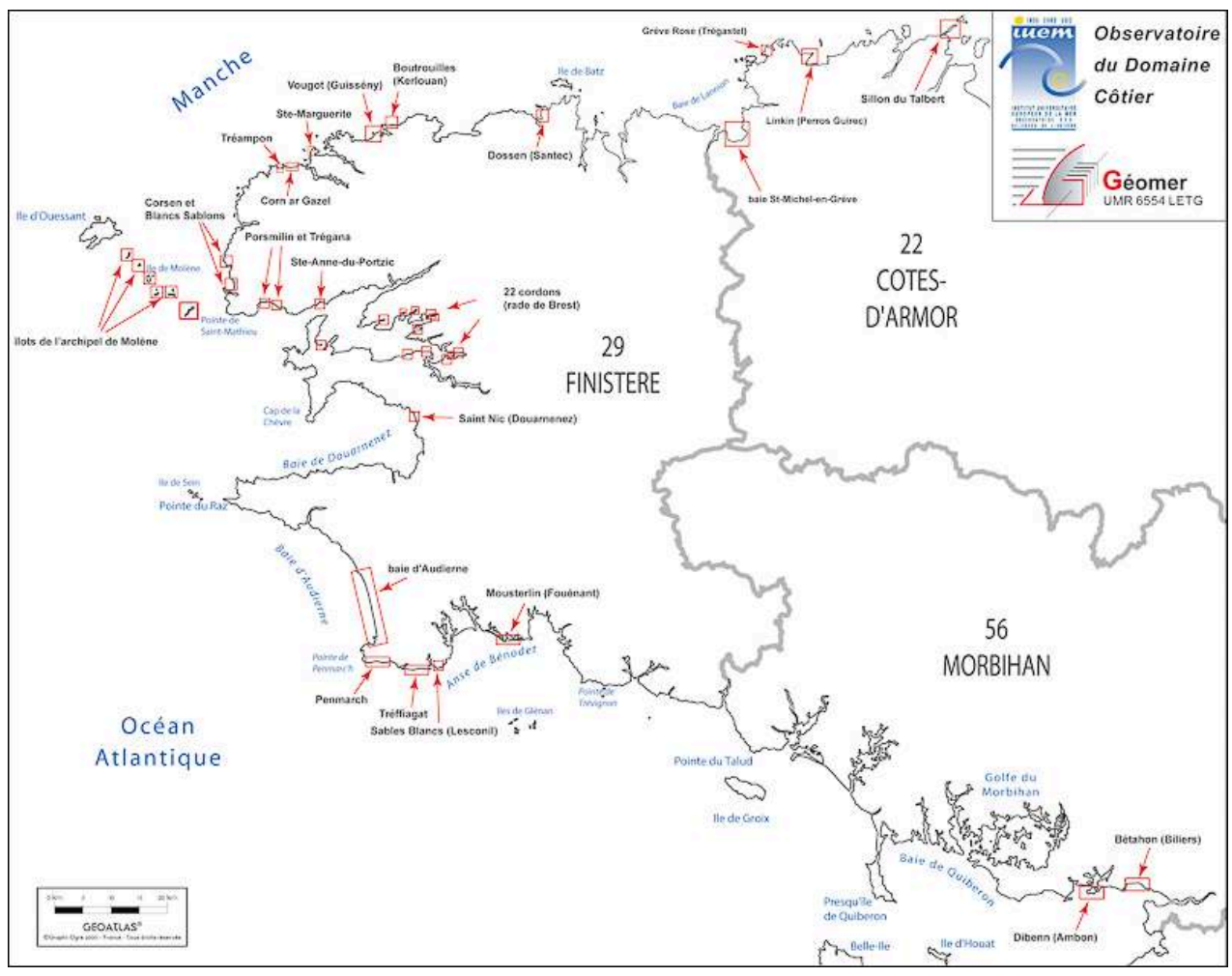

\section{Objectifs et types de mesure effectués}

31 L'objectif initial de ce suivi était en lien direct avec la recherche fondamentale ; il s'agissait de détecter un signal des variations climatiques et/ou météorologiques à partir des changements morphologiques et sédimentaires à long terme de la frange littorale. Aujourd'hui, le but de ce suivi est également de répondre à une demande sociétale en matière de recherche appliquée, et ce d'autant plus que plus de $70 \%$ de ces opérations de suivi sont financées par des collectivités. Il s'agit de constituer des séries 
de données sur l'évolution et la morphologie du trait de côte permettant d'accompagner les acteurs locaux dans les politiques de gestion et d'aménagement de l'espace littoral. Un dernier objectif consiste enfin à produire des séries de données suffisamment longues et pertinentes pour la calibration des modèles existants dans le domaine de l'ingénierie (modèles 2D et 3D sur l'évolution des plages).

Le suivi repose sur l'acquisition de trois types de levés topo-morphologiques qui sont réalisés en fonction de la morphologie du littoral :

- mesure de profils de plage supra et intertidale le long de radiale(s) perpendiculaire(s) au trait de côte, offrant une vision en deux dimensions des changements altitudinaux d'une plage. Ce type de levé le plus commun est le plus facile à mettre en œuvre quelle que soit la morphologie de la plage ;

- mesure du trait de côte obtenu par photo-interprétation pour les périodes les plus anciennes, et par mesure de terrain pour l'actuel. La limite de référence généralement retenue pour définir la position du trait de côte correspond à la limite entre la végétation dunaire et les sables vifs de haut de plage (ce type de levé n'est donc possible que lorsque la plage présente une morphologie dunaire bien marquée). Cette information offre une vision en deux dimensions des changements en plan de la ligne de rivage (cinématique du trait de côte) ;

- levés surfaciques permettant de restituer la topographie littorale en 3D à partir de Modèle(s) Numérique(s) de Terrain (MNT). Cela concerne aussi bien la mesure en milieu continental, qu'en milieu sous-marin. D'un point de vue logistique, ce type de levé demande une mise en œuvre beaucoup plus longue que les précédents.

Tous les levés de terrain en milieu supralittoral et intertidal sont effectués au DGPS suivant un protocole rigoureux qui permet de récupérer une information géoréférencée et directement rattachée au réseau géodésique français (Suanez et al., 2008). Les mesures bathymétriques sont effectuées à partir d'un sondeur multifaisceaux embarqué permettant aussi de récupérer une information géoréférencée et raccordée au système géodésique français. La fréquence d'échantillonnage dépend de la problématique que l'on s'est fixée. Une fréquence haute (1 levé par mois) est généralement retenue afin d'analyser l'impact morphogénique des évènements tempétueux sur le système littoral. Une fréquence plus basse (1 levé par an) est adoptée pour la simple mesure de l'évolution du bilan sédimentaire des cordons littoraux.

\section{Archivage, valorisation et affichage des données}

L'archivage des données est actuellement réalisé au sein d'un système d'informations appelé MEnIr (Mémoire Environnementale Iroise) qui a été développé par le laboratoire Géomer (Gourmelon et al., 2005). Ce système permet entre autres de stocker des données géoréférencées ainsi que les fiches descriptives de métadonnées les décrivant ${ }^{5}$. Lors de sa conception, la norme ISO 19115 de catalogage des métadonnées pour l'information géographique venait d'être publiée et son implémentation dans MEnIr avait été envisagée. Cependant, sa relative complexité liée au nombre de champs trop important ainsi que des retours mitigés des utilisateurs et producteurs de données, nous ont poussé à établir une liste de champs de métadonnées propre à nos besoins. Ainsi, l'interopérabilité de MEnIr avec d'autres systèmes de catalogage a été fortement diminuée.

Plus récemment, un portail numérique a été développé afin de permettre l'affichage, la valorisation et la mise à disposition des données acquises spécifiquement dans le cadre 
de l'observatoire du trait de côte. Cette interface Web appelée MADDOG (Mise A Disposition de Données d'Observation Géomorphologique), est directement accessible via Internet ${ }^{6}$. L'architecture et les fonctionnalités générales de MADDOG sont décrites dans l'illustration 5.

Illustration 5 - Architecture technique et fonctionnement du portail MADDOG

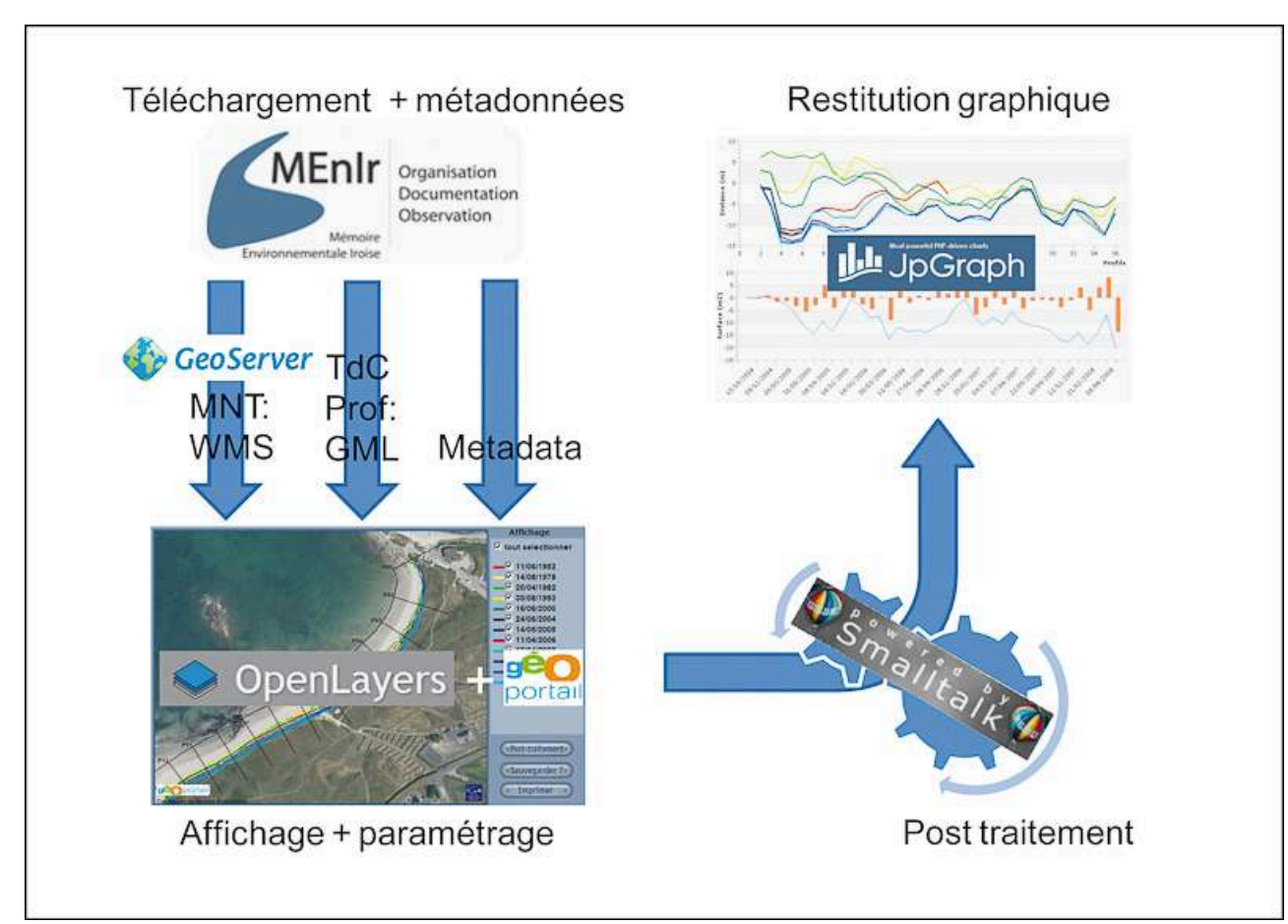

Dans un premier temps, les données topo-morphologiques relatives à l'observation du trait de côte sont stockées (en format ASCII) et cataloguées dans MEnIr; ce sont de simples fichiers .txt compilant la position des points topographiques en coordonnées $\mathrm{X}$, $\mathrm{Y}, \mathrm{Z}$. Le catalogage est réalisé sous la forme de fiches de métadonnées dans lesquelles se trouvent toutes les informations relatives à l'acquisition, à la qualité, au format et à la propriété de la donnée. La mise à disposition des données archivées se fait par l'intermédiaire du catalogue.

Dans un second temps le portail MADDOG vient interroger la base de données MEnIr afin que les mises à jour se fassent automatiquement lorsque de nouveaux jeux de données ont été ajoutés. L'affichage et la sélection des sites géographiques où la donnée existe se fait par l'intermédiaire d'une carte dynamique interactive générée à l'aide dela librairie javascript OpenLayer ${ }^{7}$ et del' API géoportail ${ }^{8}$ de l'IGN. Une fois le site sélectionné, les données topo-morphologiques relatives au trait de côte et au profil de plage nouvellement ajoutées sont ensuite converties au format standardisé et interopérable $\mathrm{GML}^{9}$, puis affichées sur la carte dynamique. Les données topomorphologiques surfaciques de type MNT (semis de points des relevés) sont diffusées à partir d'un flux $W M S^{10}$ via un geoserver ${ }^{11}$ avant d'être affichées sur la carte dynamique sous la forme d'un semis de points. L'interface Web MADDOG permet enfin de générer du post-traitement de moyenne complexité afin d'obtenir une information sur les évolutions morphologiques sous la forme de sérialisation temporelle pour la cinématique du trait de côte ou de calculs de volumes sédimentaires pour les MNT et les profils de plage. Ces traitements se font on line en fonction du paramétrage effectué 
par l'utilisateur et font appel à un script SmallTalk ${ }^{12}$. Les résultats sont enfin restitués sous forme de graphiques à l'aide de la librairie PHPJpGraph ${ }^{13}$.

\section{Conclusion} et 4 . En effet, ces suivis sont à l'heure actuelle ceux qui répondent au mieux à la définition "d'observatoire " telle que définie par l'INSU tant du point de vue des protocoles de mesures mis en place que de l'archivage et de la valorisation des données acquises. De même, ce sont ceux qui couvrent des aires géographiques les plus cohérentes allant du département à la région. Les observatoires de type 1 et 2 , qui répondent le plus souvent à des demandes bien spécifiques, ne serviraient alors qu'à produire de la donnée ponctuelle sur des espaces géographiques plus restreints et à des fréquences plus importantes; pour autant, il importe que ces données puissent être intégrées aux longues séries acquises par les observatoires de type 3 et 4 . Dans le même temps, il apparaît nécessaire de développer l'interopérabilité de l'information de manière à produire une image exhaustive de l'évolution à long terme du trait de côte en France métropolitaine et dans les départements d'Outre-mer. Ces deux actions prioritaires de structuration à l'échelle nationale et d'interopérabilité des données permettraient d'aboutir à une vision homogène des évolutions pour tout le littoral français, de comparer les secteurs entre eux, et de prioriser les actions de gestion et/ou de défense du trait de côte à engager.

Si la structuration des observatoires à l'échelle nationale relève avant tout de l'action politique de l'État, l'interopérabilité des données repose sur un travail de mise aux normes qui peut être réalisé indépendamment par chacun des maîtres d'œuvre, notamment par l'application de la directive INSPIRE. Le portail MADDOG est en partie conforme à cette directive de par l'accessibilité des métadonnées et des données ainsi que par l'effort de standardisation des formats de données (GML et WMS). Cependant le catalogue de métadonnées MEnIr n'est pas à la norme ISO 19115 préconisée par la directive INSPIRE. Les projets d'évolution en cours du portail visent dans un premier temps à se mettre en conformité avec cette dernière en établissant une véritable IDS ${ }^{14}$ basée sur des outils interopérables (GeoNetwork pour les métadonnées, GeoServer pour les données), et dans un second temps, dans une optique plus recherche et développement $(R \& D)$, à rendre génériques les processus de post-traitements à l'aide du standard $W P S^{15}$. 


\section{BIBLIOGRAPHIE}

Aubié S., Mugica J., Mallet C., Hoareau A., 2011. Observatoire de la Côte Aquitaine. Bilan des activités réalisées dans le cadre de la convention 2009-2010. BRGM/RP-59259-FR, 80 p., 34 illustrations, 2 tableaux et 1 annexe.

Barras A.V., Koller M.-M., 2010. État du littoral de la Martinique un an après le cyclone DEAN (17 août 2007) et suite à l'épisode de houle cyclonique OMAR (16 octobre 2008). Rapport BRGM/RP-57555-FR, 61 p., 48 illustrations, 4 annexes hors rapport.

BCEOM, 2007. Étude de l'évolution du trait de côte du littoral des Alpes-Maritimes. Résumé non technique, Février 2007. MAR N60554C, 6 p.

Bersani C., Simoni, M.-L., Allain Y.-M., Ribière G., Denègre J., Planques P., Tugayé Y., 2006. Rapport relatif au schéma d'organisation des dispositifs de recueil de données et d'observation sur le littoral. Ministère de l'Intérieur et de l'Aménagement du Territoire (Inspection générale de l'administration), Ministère des Transports, de l'Equipement, du Tourisme et de la Mer (Conseil Général des Ponts et Chaussées), Ministère de l'Écologie et du Développement Durable (Inspection Générale de l'Environnement), $120 \mathrm{p}$

Birkemeier W., 1985. Field data on seaward limit of profil change. Journal of Waterway, Port, Coastal and Ocean Engineerineg, vol. 111, n 3, p. 598-602.

Bulteau T., Garcin M., Oliveros C., Lenôtre N. 2011. Synthèse des travaux menés sur l'observation de l'évolution du trait de côte. Rapport BRGM/RP-59396-FR, 136 p.

Colas S., 2006. Le quart du littoral recule du fait de l'érosion. Le 4 pages Ifen, $\mathrm{n}^{\circ} 113$, (Institut Français de l'Environnement), p. 1-4.

Dehouck A., Cuq V., Fichaut B., Suanez S., 2004. Premiers éléments de caractérisation morphodynamique de deux plages de la mer d'Iroise. Actes du colloque des VIII ${ }^{\mathrm{es}}$ Journées Nationales Génie Côtier - Génie Civil, Compiègne, 7-9 septembre 2004, D. Levacher, P. Sergent, A. Ouahsine et le Centre Français du Littoral (Eds.), Tome 1, p. 357-364.

European Commission, 2004. Living with Coastal Erosion in Europe: Sediment and Space for Sustainability. Luxembourg, Office for Official Publications of the European Communities, Part I "Major findings and Policy Recommendations of the EUROSION project", 10 May 2004, 54 p.; Part II "Maps and statistics", 29 May 2004, 25 p.

Favennec J., Mallet C., 2008. Un atlas de l'érosion marine réalisé dans le cadre de l'observatoire de la côte pour guider les choix d'aménagement de la côte sableuse d'Aquitaine. Actes du colloque international pluridisciplinaire « Le littoral : subir, dire, agir », Lille (France), 16-18 janvier 2008 http:// www.meshs.fr/documents/pdf/publications/actes/colloque_littoral/Favennec.pdf)

Fichaut B., Suanez S., 2005. Mise en place d'un suivi morphosédimentaire dans l'archipel de Molène : exemple de l'îlot de Trielen. In Milieux littoraux. Nouvelles perspectives d'étude, Durand P., Goeldner-Gianella L. (dir.), Ed. L'Harmattan, p. 53-74.

Gourmelon F., Lafaye J.-Y., Rouan M., Faucher C., 2005. MENIR : mémoire environnementale Iroise. Actes du colloque de bilan du programme interdiciplinaire "société de l'Information" 2001-2005 Actes du colloque de bilan du programme interdisciplinaire "Société de l'Information » 2001-2005, Lyon : France (2005), http://hal.archives-ouvertes.fr/hal-00082929/fr/ 
Hamm L., Capobianco M., Dette H.-H., Lechuga A., Spanhoff R., Stive M.J.F., 2002. A summary of European experience with shore nourishment. Coastal Engineering, vol. 47, n 2, p. 237-264.

Howd P.-A., Birkemeir W.-A., 1987. Beach and nearshore survey data: 1981-1984, CERC Field Research Facility: Technical Report CERC-87-9. U.S. Army Waterways Experiment Station, Vicksburg, Mississippi, (non paginé).

IN VIVO, 2004. Évolution du trait de côte du littoral varois. Rapport définitif, novembre 2004, 139 p.

Koster M.-J., Hillen, R., 1995. Combat erosion by law: coastal defence policy for The Netherlands. Journal of Coastal Research, vol. 11, $\mathrm{n}^{\circ}$ 4, p. 1221-1228.

Lachassagne P., Paulin C., 1990. Dynamique actuelle des côtes de la Guadeloupe et de ses dépendancesInventaire des zones d'évolution et définition des travaux nécessaires à une meilleure connaissance et gestion du littoral guadeloupéen. Rapport BRGM/RP-31176-FR, 21 p., 1 figure et 6 annexes.

Larson M., Krauc N.-C., 1994. Temporal and spatial scales of beach profil change, Duck, North Carolina. Marine Geology, vol. 117, n 1-4, p. 75-94.

Leatherman, S.-P., 2003. Shoreline change mapping and management along the U.S. East coast. Journal of Coastal Research, Special Issue n 38, p. 5-13.

Lee G.-h., Birkemeir W.-A., 1993. Beach and nearshore survey data: 1985-1991, CERC Field Research Facility: Technical Report CERC-93-3. U.S. Army Waterways Experiment Station, Vicksburg, Mississippi, 26 p. plus appendices.

Louisse C.-J., van der Meulen F., 1991. Future coastal defence in the Netherland: strategies for protection and sustainable development. Journal of Coastal Research, vol. 7, n 4,1027-1041.

Mason C., 1985. Recent R\&D accomplishments at the coastal engineering research center field research facility. Oceans, vol. 17, p. 78-84.

Meur-Férec C., Deboudt, P., Morel V. 2008. Coastal risks in France: an integrated method for evaluating vulnerability. Journal of Coastal Research, vol. 24, n² 2B, p. 178-189.

Mugica J., Mallet C., Aubié S., Hoareau A., Pierson J., 2009. Observatoire de la Côté Aquitaine. Bilan des activités réalisées dans le cadre de la convention 2007-2008. BRGM/RP-57071-FR, 66 p. 19 illustrations, 2 tableaux et 1 annexe.

Reeve D., Li Y., Lark M., Simmonds D., 2007. An investigation of the multi-scale temporal variability of beach profiles at Duck using wavelet pasket transforms. Coastal Engineering, vol. 54, $\mathrm{n}^{\circ}$ 5, p. 401-415.

Rieb G., Walker P., 2001. Suivi morphologique du littoral. Géologues, n 129, p. 75-79.

Ruggiero P., Voigt B., Kaminsky G., 2000. Beach monitoring for enhanced decision-making. Coastal Society 17th Conference Coasts at the Millennium, 9-12 July 2000, Portland, Oregon, (non paginé).

SAFEGE, 2008. Étude de l'évolution du trait de côte du littoral des Bouches-du-Rhône au regard de l'érosion marine. Présentation dans le cadre de la réunion plénière Pôle métier Mer/littoral CRIGE-PACA du 25 septembre 2008.

Solomon S.-M., Forbes D.-L., 1999. Coastal hazards and associated management issues on South Pacific Islands. Ocean \& Coastal Management, vol. 42, $\mathrm{n}^{\circ}$ 6-7, p. 523-554.

Southgate H.-N., 2008. Data-based forecasting of beach volumes on monthly to yearly timescales. Coastal Engineering, vol. 55, n 12, p. 1005-1015.

Suanez S., Dehouck A., Stéphan P., 2008. Incertitude de la mesure de terrain en géomorphologie littorale. Approche statistique et quantification des marges d'erreur. In Incertitude et 
environnement. La fin des certitudes scientifiques, Allard P., Fox D., Picon B. (dir.), Aix-en-Provence, Edition EDISUD, Collection Ecologie Humaine, p. 127-139.

Suanez S., Cariolet J.-M., Fichaut B., 2010. Monitoring of Recent Morphological Changes of the Dune of Vougot Beach (Brittany, France) Using Differential GPS. Shore \& Beach, vol. 78, $\mathrm{n}^{\circ} 1$, p. 37-47.

\section{NOTES}

1. http://www.caribsat.com

2. http://www.interreg-caraibes.org

3. La directive Inspire a été publiée au Journal officiel des Communautés européennes (JOCE) le 25 avril 2007, et est entrée en vigueur le 15 mai 2007. Le premier article insiste sur la nécessité de fixer les règles pour «établir l'infrastructure d'information géographique dans la Communauté européenne, aux fins des politiques environnementales communautaires et des politiques ou activités de la Communauté susceptibles d'avoir une incidence sur l'environnement ». http://inspire.jrc.ec.europa.eu/

4. http://www-iuem.univ-brest.fr/observatoire/le-domaine-cotier/

5. http://menir.univ-brest.fr/observation/idfix/consulting.php

6. http://menir.univ-brest.fr/maddog/

7. http://openlayers.org/

8. https://api.ign.fr/geoportail/index.do

9. http://www.opengeospatial.org/standards/gml

10. http://www.opengeospatial.org/standards/wms

11. http://geoserver.org/

12. http://www.smalltalk.org/

13. http://jpgraph.net/

14. Infrastructure de Données Spatiales.

15. http://www.opengeospatial.org/standards/wps

\section{RÉSUMÉS}

En préalable à l'engagement $74 \mathrm{du}$ Grenelle de la mer « développer une méthodologie et une stratégie nationale pour la gestion du trait de côte, pour le recul stratégique et la défense contre la mer [...]», une synthèse des travaux menés sur l'observation du trait de côte en France métropolitaine et dans les DOM a été réalisée par le BRGM à la demande du MEEDDM. Les conclusions de cette étude insistent sur la très forte hétérogénéité des observatoires allant du suivi ponctuel et limité dans l'espace, à des suivis pérennes régionaux impliquant de nombreux acteurs. Ces différents cas de figure permettent d'établir une typologie en quatre classes des différents types d'observatoire. Ce travail insiste également sur la diversité des maîtres d'oeuvre en charge de ces "observatoires", ayant pour conséquence une hétérogénéité des méthodes et des protocoles d'acquisition des données. De ce fait, la comparaison des informations d'un observatoire à l'autre n'est pas toujours aisée. De même, les diverses modalités de stockage de cette information indiquent que d'un opérateur à l'autre, l'interopérabilité des métadonnées 
reste faible. Comme le montre l'étude de cas qui est traitée en fin d'article (l'observatoire du Domaine Côtier de l'IUEM), la mise aux normes des données acquises suivant la directive européenne INSPIRE, accompagnée d'une meilleure structuration et d'une pérennisation des suivis, apparaissent nécessaire. Cela favoriserait les possibilités d'échange et permettrait d'obtenir une vision claire de l'évolution du trait de côte en France métropolitaine et dans les départements d'Outre-mer sur le long terme.

In pre-commitment of $74 \mathrm{du}$ Grenelle de la mer "develop a methodology and a national strategy for shoreline management, the strategic retreat and defense against the sea [...]," a synthesis of work on observatories of shoreline in mainland France and overseas departments was carried out by BRGM at the request of MEEDDM. The findings of this study emphasize the strong heterogeneity of surveys from short time monitoring and limited in space, to long term monitoring at regional scale involving many actors. These different scenarios provide a typology of four classes of different types of observatory. This work also highlights the diversity of actors in charge of these surveys, resulting in a diversity of methods and protocols for data acquisition. Thus, the comparison of information from one observatory to another is not always easy. Similarly, various forms of storage of this information indicate that one actor to another, the interoperability of metadata remains low. As shown in the case study is discussed at the end of article (l'observatoire $d u$ Domaine Côtier de l'IUEM), the upgrading of the data acquired after the European INSPIRE directive, along with a better structure and to a continuation of monitoring, appear necessary. This would promote opportunities for exchange and would provide a clear vision of the long term evolution of the shoreline in France and the overseas departments.

\section{INDEX}

Mots-clés : interopérabilité, métadonnées, observatoire, trait de côte, typologie

Keywords : interoperability, metadata, observatory, shoreline, typology

Thèmes : Sur le Champ - Sur le Terrain

\section{AUTEURS}

\section{SERGE SUANEZ}

Serge Suanez, serge.suanez@univ-brest.fr, est maître de conférences à l'Université de Brest, chercheur au laboratoire LETG-Géomer-Brest - UMR 6554 CNRS, Institut Universitaire Européen de la Mer. Il a publié récemment :

- Suanez S., Fichaut B., Magne R., Ardhuin F., Corman D., Stéphan P., Cariolet J.-M., 2011. Changements morphologiques et bilan sédimentaire des formes fuyantes en queue de comète de l'archipel de Molène (Bretagne, France). Géomorphologie : relief, processus, environnement, $\mathrm{n}^{\circ} 2$, p. 187-204.

- Suanez S., Stéphan P., 2011. Natural and Human Forcing on the Mesoscale Shoreline Dynamics of Saint-Michel-en-Grève Bay (Brittany, France). Shore \& Beach, vol. 79, n² 2, p. 19-38.

\section{MANUEL GARCIN}

Manuel Garcin, m.garcin@brgm.fr , est ingénieur au BRGM, Service Risques Naturels - Unité Risques côtiers. 


\section{THOMAS BULTEAU}

Thomas Bulteau, t.bulteau@brgm.fr, est ingénieur au BRGM, Service Risques Naturels - Unité Risques côtiers.

\section{MATHIAS ROUAN}

Mathias Rouan, mathias.rouan@univ-brest.fr, est ingénieur CNRS au laboratoire LETG-GéomerBrest - UMR 6554 CNRS, Institut Universitaire Européen de la Mer. Il a publié :

- Faucher C., Gourmelon F., Lafaye JY, Rouan M., 2009. Mise en œuvre d'une mémoire environnementale adaptée aux besoins d'un observatoire du domaine côtier : MEnIr. Revue Internationale de Géomatique, vol. 19, n 1, p. 7-26.

\section{LOÏC LAGADEC}

Loïc Lagadec, Loic.lagadec@univ-brest.fr, est maître de conférences à l'Université de Brest, chercheur au Lab-STICC, UMR 3192 CNRS, Département d'Informatique, UFR Sciences et Techniques.

\section{LAURENCE DAVID}

Laurence David, laurence.david@univ-brest.fr, est ingénieur CNRS au laboratoire LETG-GéomerBrest - UMR 6554 CNRS, Institut Universitaire Européen de la Mer. 